\title{
Individual differences in field independence influence the ability to determine accurate needle angles
}

\author{
Ahmad Y. Sheikh, MD, , Madeleine Keehner, PhD, ${ }^{\mathrm{b}}$ Audrey Walker, BS, ${ }^{\mathrm{b}}$ Paul A. Chang, BS, ${ }^{\mathrm{a}}$ \\ Thomas A. Burdon, MD, and James I. Fann, MD $^{\mathrm{a}}$
}

Objective: "Field dependence" is used in cognitive psychology to describe an individual's tendency to be visually distracted by the surrounding environment. Notwithstanding the role of field dependence in contexts in which spatial judgment is important, such as piloting an aircraft, to date, studies linking field dependence to surgical skills have been limited. We evaluated whether field dependence correlates with an ability to anticipate appropriate needle angles in a simulated setting.

\begin{abstract}
Methods: Trainees underwent field dependence testing and then participated in a surgical skills exercise. Correlations between field dependence and surgical skill were computed. Specifically, cardiothoracic surgery residents $(\mathrm{n}=11)$ took a battery of cognitive examinations to assess general reasoning and visuospatial judgment. Two written tests, the Rod and Frame test and the Judgment of Line Orientation test, measured the degree of field dependence. The subjects then underwent surgical skills testing. Using a standard needle driver, the participants placed curved needles into a cylindrical silicone mitral valve model with 10 premarked needle entry and exit sites. The components assessed included the ability to load a needle on driver at the appropriate angle.
\end{abstract}

Results: The test results showed a parametric distribution, with internal cognitive testing controls demonstrating valid testing techniques and methods. Performance on the cognitive tests measuring spatial judgment and field dependence correlated significantly with skill at determining the appropriate needle angle load in an inverse fashion (Judgment of Line Orientation test, $r=0.61, P<.05$; Rod and Frame test, $r=-0.52, P=.05$ ), suggesting that residents who were not distracted by surrounding objects performed better. Performance on the cognitive examinations did not correlate with resident training level.

Conclusions: Although our study was of a small cohort, the findings suggest that individuals described as field independent (not easily distracted by external visual cues) might possess improved ability to determine appropriate needle angle loads compared with field-dependent individuals. Additional studies examining the role field dependence might play in the acquisition and execution of surgical tasks are warranted. (J Thorac Cardiovasc Surg 2014;148:1804-10)

See related commentary on page 1811 .

In recent years, medical education has increasingly shifted toward competency-based learning with usage of simulation in surgical and nonsurgical disciplines. ${ }^{1-6}$

From the Department of Cardiothoracic Surgery, ${ }^{a}$ Stanford University, Stanford, Calif, and the Veterans Affairs Palo Alto Health Care System, Palo Alto, Calif; and University of Dundee, ${ }^{b}$ Dundee, United Kingdom.

The present study was supported by the an educational award from The Western Thoracic Surgical Association (Doty Award) and simulation grants from the Thoracic Surgery Foundation for Research and Education and the Center for Immersive and Simulation-based Learning at Stanford University.

Disclosures: Ahmad Y. Sheikh reports consulting fees from Aginity. All other authors have nothing to disclose with regard to commercial support.

Read at the 39th Annual Meeting of The Western Thoracic Surgical Association, Coeur d'Alene, Idaho, June 26-29, 2013.

Received for publication June 25, 2013; revisions received March 20, 2014; accepted for publication May 2, 2014; available ahead of print June 10, 2014.

Address for reprints: James I. Fann, MD, Department of Cardiothoracic Surgery, Stanford University, 300 Pasteur Dr, Stanford, CA 94305 (E-mail: jfann@ stanford.edu).

0022-5223/\$0.00

Published by Elsevier Inc. on behalf of The American Association for Thoracic Surgery

http://dx.doi.org/10.1016/j.jtcvs.2014.05.008
Although this shift has prompted a surge in the use of simulation-based training by surgical programs, a paucity of investigation exists regarding the cognitive basis of how surgical skills are acquired in simulated or "real" environments. Effective training will likely require training in surgical environments and an understanding of the cognitive paradigms to best instruct individuals in mastering the necessary psychomotor skills.

Because the published data regarding the learning processes in surgical education are limited, it would be important to provide a link between surgical skills acquisition and cognitive psychology. We selected a well-known cognitive phenotype that requires spatial judgment, namely field dependence, which classifies an individual's ability to focus on a specific visuomotor task in the setting of "distractors." Subjects can have varying degrees of field dependence, with those who are more field dependent more distracted by the environment. Field dependence, a subset of spatial judgment, differs from the concept of spatial visualization. ${ }^{7}$ Taken together, the areas of spatial judgment and spatial visualization can characterize how individuals process objects in 3 


\section{Abbreviations and Acronyms \\ $3 \mathrm{D}=3$-dimensional \\ $\mathrm{NL}=$ load of needle on driver}

dimensions. We chose to focus on spatial judgment and, more specifically, field dependence, because this is most appropriate to the tasks in cardiothoracic surgery. For example, a surgeon often needs to judge the depth of the working field, the relationship between anatomic structures, and how instruments should be best positioned to easily place a suture. In contrast, spatial visualization focuses more on how individuals perceive and manipulate objects in 3 dimensions, a process that might be more useful for individuals, such as structural biochemists, who are required to mentally rotate objects in space.

Because operating in the thorax provides spatial constraints that limit the users' degrees of freedom, the appropriate selection of needle angles achieved by correctly loading needles onto a needle driver should be considered an essential skill. In particular, 3 points can be emphasized: (1) that the needle enters orthogonal to the tissue plane; (2) that the subject causes minimal distortion to the surrounding tissue as the needle is pushed through its arc; and (3) that the subject follows the curve of the needle as it is drawn out of the tissue to minimize the trauma induced by the needle tract. In the present initial study, we evaluated whether field dependence (ie, an ability to assess the spatial orientation of the object of interest in the setting of surrounding distractors) correlated with an ability to anticipate appropriate needle angles in a simulated setting.

\section{METHODS}

Eleven cardiothoracic surgery residents ( 9 men, 2 women) participated in the present study. Of the 11 residents, 4 had previously been trained in a traditional, 5-year general surgery program, and 7 were in the integrated 6 -year cardiothoracic surgery program. All participants at a postgraduate year training level of $\geq 4$ had performed cardiac valve operations in the clinical setting. The first- to third-year integrated residents had not performed cardiac surgery operations except in the simulation laboratory. The institutional review board approved the present study.

\section{Protocol}

The residents underwent a battery of 5 standardized cognitive tests designed to assess general reasoning, spatial visualization, and spatial judgment. Field dependence, the primary focus of our study, is within the category of spatial judgment skill. Using a standard needle driver and cylindrical silicone mitral valve model, the residents completed 10 needle "swings" at preselected positions.

\section{Cognitive Tests}

General reasoning. Raven's Advanced Progressive Matrices (12 items, 12 minutes) is an abstract reasoning, nonverbal, assessment of the capacity to determine logical relationships among objects. Each item shows a series of patterns connected by a logical relationship, and the test taker must decide which pattern would come next in the sequence.
Raven's test has been claimed to capture cognitive processes analogous to general fluid intelligence or complex working memory capacity. This cognitive test served to validate the entire written assessment and served as in internal control to ensure that the results from our testing were free of learner bias or practice effect.

Spatial visualization. We administered 2 spatial visualization tests: the Mental Rotations test (40 items, 6 minutes) and an adapted version of the Purdue Visualization of Views test ( 24 items, 8 minutes). Each mental rotation item shows a drawing of a 3-dimensional (3D) object. The task is to decide which 2 of 4 alternate objects represent the same target object rotated into a different orientation. The mental rotation test is designed to evaluate the ability to mentally rotate objects. Each visualization of the test item shows a drawing of a glass cube with a 3D object floating in its center. Beneath is the same 3D object drawn in a different orientation. The task is to decide from which corner of the glass cube one must look to see the object in the alternate view. This test is designed to evaluate the ability to imagine objects from alternate viewpoints. These 2 tests are considered to require many of the same complex mental spatial visualization processes. However, these tests do not represent field dependence. We included them in the present study to ensure we were observing phenomena specific to the trait of field dependence and not general spatial visualization.

Spatial judgment. We selected 2 spatial judgment tests that would capture the degree of an individual's field dependence: the Judgment of Line Orientation test (18 items, 2 minutes) and a paper and pencil version of the Rod and Frame test (9 items, no time limit), adapted from Cote and O'Donnell. ${ }^{10}$ Each item in the Judgment of Line Orientation test presents a target line in a given orientation. The task is to select the matching orientation from 21 possible lines, which are arranged in a group and encompass a range of orientations from which only the correct one must be chosen. This test assesses the skill of finding a match between the angles of 2 lines separated in space when several plausible distractors are nearby from which to choose.

The adapted Rod and Frame test shows a linear series of dots indicating a straight line at a given angle. The line of dots is presented within a square frame, which can be parallel to, or tilted in relation to, the paper on which it has been printed. The task is to replicate the line of dots by drawing them as accurately as possible inside a copy of the upright or tilted frame. This test was designed to evaluate the ability to mentally separate a stimulus from potentially misleading or biasing surrounding cues.

Both the Rod and Frame test and the Judgment of Line Orientation test are considered primarily perceptual tests, rather than tests of visualization, because they do not require complex manipulation of spatial mental representations (in contrast to spatial visualization tests). Thus, they require the test taker to accurately perceive, mentally encode, and "read out" the spatial information to identify or generate matching angles. Both tests involve angle judgment in the face of potentially distracting elements in the nearby space that must be disregarded or correctly rejected. Thus, both tests are assumed to test field independence. Sample questions assessing spatial visualization and spatial judgment are shown in Figure 1.

\section{Technical Skills Assessment}

"Plastic" mitral valve model. The mitral valve model is a silicone-based cylinder with an opening of $7 \mathrm{~cm}$ in diameter and a depth of $5.5 \mathrm{~cm}$ (Chamberlain Group, Great Barrington, Mass; Figure 2). The commissure-to-commissure distance is $35 \mathrm{~mm}$, and the anteroposterior dimension measures $26 \mathrm{~mm}$. The cylindrical configuration and the relatively flat plane of the annulus require awareness of the needle angles to optimally place the sutures. The annulus provides a reasonable tactile response to suture placement, permitting assessment of the depth of suture placement and the spacing between the sutures. Target sites were marked on the mitral valve models using black and red indelible ink to indicate the entry and exit sites, respectively, for right-hand individuals and the reverse for left-hand individuals. Standard surgical instruments were used. The sutures 



FIGURE 1. A panel of sample questions designed to assess spatial visualization and spatial judgment. The correct answers for each sample question are highlighted in red. A, The Visualization of Views test depicts a 3-dimensional (3D) object floating within a glass cube and the same object from a different vantage point immediately below. The trainee must identify which corner of the cube would provide the vantage to generate the bottom view of the object. B, The Mental Rotations test shows a drawing of a 3D object. The subject must decide which 2 of 4 alternate objects represent the same target object rotated to a different orientation. C, The Judgment of Line Orientation test. Each item presents a target line in a given orientation. The subject selects the matching orientation from 21 possible lines, which are arranged in a group and encompass a range of orientations from which only the correct one must be chosen. The correct answers for each line orientation are labeled in color and circled below. D, The adapted Rod and Frame test shows a linear series of dots indicating a straight line. The line of dots is presented within a square frame, which can be parallel to, or tilted in relation to, the paper on which it has been printed. The task is to replicate the line of dots by drawing them as accurately as possible inside a copy of the upright or tilted frame.

were 2-0 Ethibond with RB-1 needles (Ethicon, Inc, Somerville, NJ). The procedure was video-recorded using a high-definition video camera.

Performance assessment. All the data were de-identified and stored on a computer hard disk. Video recordings were submitted to 2 senior faculty surgeons who were unaware of the identity of the residents and their test scores.
Resident performance was evaluated by a review of the video recordings, and the score for each component of the assessment was determined by a consensus between the 2 surgeons. Resident performance was assessed using a 5-point rating scale, modified from the Objective Structured Assessment of Technical Skills described by Reznick and $\mathrm{MacRae}^{2}$ (Figure 3).
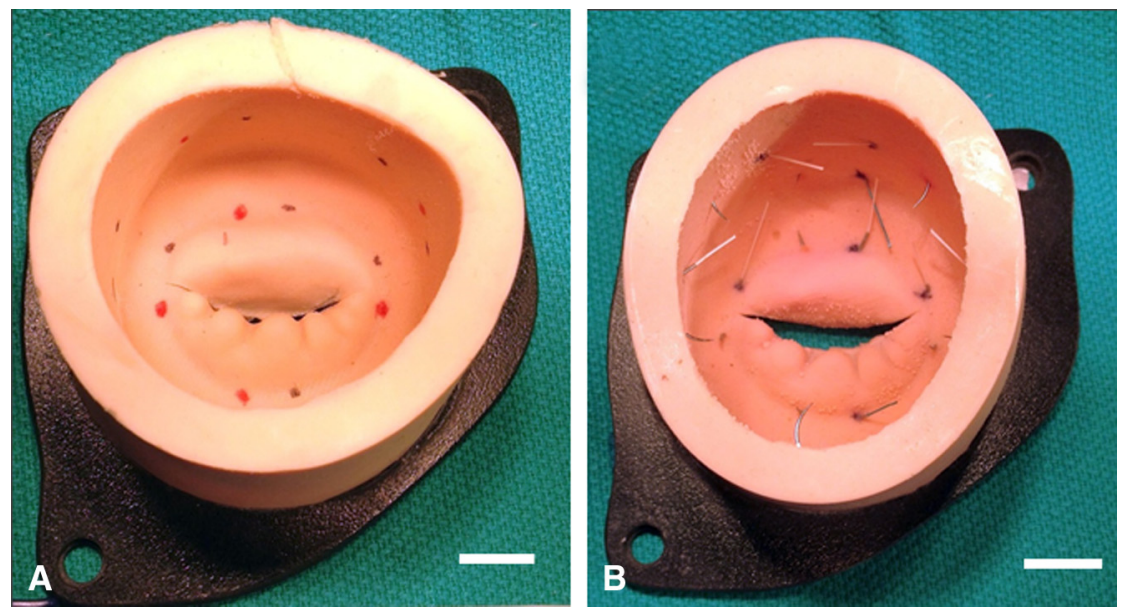

FIGURE 2. The simulation system to determine the trainee's skill in anticipating and correctly deploying the needle angles. A, The cylindrical mitral valve cylinder was used, with the target entry and exit sites marked with red and black dots, respectively. B, Valve model after completed the exercise with the needles left in place. All performances were recorded by video for later review by senior surgeons-evaluators. Scale bar $=1 \mathrm{~cm}$. 




YR OF TRAINING

$\begin{array}{lc} & \text { Excellent } \\ 3 & 5 \\ \text { Moderate hesitation } & \text { No hesitation } \\ \text { Few trial passes } & \text { No trial passes } \\ \text { Some reloading of needle } & \text { Few or no reloading of needle } \\ \text { Partial adjustment for space/depth } & \text { Correct adjustment for space/depth }\end{array}$

Additional Comments:

\begin{tabular}{clllll}
\hline $\begin{array}{c}\text { 2. Point of needle } \\
\text { orthogonal to target }\end{array}$ & 1 & 2 & 3 & 4 & 5 \\
Markedly tangential & Some tangential & & Orthogonal
\end{tabular}

Additional Comments:

\begin{tabular}{|c|c|c|c|}
\hline 3. Distortion of cylinder & 2 & 3 & 5 \\
\hline by the needle driver & Marked distortion & Some distortion & No distortion \\
\hline in placing needle & $\begin{array}{l}\text { No able to keep in center, } \\
\text { eccentric }\end{array}$ & Slight eccentric in cylinder & Stayed in middle of cylinder \\
\hline
\end{tabular}

Additional Comments:

$\begin{array}{ll}\text { Slight eccentric in cylinder } & \text { No distortion } \\ & \text { Stayed in middle of cylinder }\end{array}$

\begin{tabular}{|c|c|c|c|}
\hline 4. Appropriate backhand & 1 & 4 & 5 \\
\hline or forehand & $\begin{array}{l}\text { No able to determine } \\
\text { forehand or backhand }\end{array}$ & Some hesitation & No hesitation \\
\hline & Not consider depth/space & Partially consider depth/space & Consider depth/space \\
\hline
\end{tabular}

Additional Comments:

5. Placement and accuracy 1

along annular plane Off target in all

2
Additional Comments:

FIGURE 3. Evaluation form for skills performance for needle angles.

The residents were rated on 5 components. The first was the load of the needle on the driver (NL). It is a global rating evaluating an individual's ability to anticipate the needle angle from the perceived entry and exit targets. The second component was whether the point of the needle was orthogonal to the target, which captures whether the needle was at a right angle orientation to the entry target. The third component, distortion of the cylinder, assessed the ability to suture within the confined space without distorting the model. The fourth, the choice of backhand or forehand, reflected an individual's ability to choose a forehand or backhand approach to drive the needle through the entry and exit targets. Finally, placement and accuracy along the annular plane evaluated the ability to properly place the needle into the selected targets at the plane of the annulus.

Statistical analysis. Comparative and descriptive statistics were calculated using the Statistical Package for Social Sciences (SPSS,
Chicago, Ill) software package for ordinal and continuous variables. The performance data from the skills assessment component are presented as the mean \pm standard deviation. Correlations between the cognitive testing scores and the expert assessment of surgical skills were obtained using Pearson's coefficient. A correlation coefficient of $r \geq 0.52$ was considered significant.

\section{RESULTS}

\section{Cognitive Testing}

The group scores for the cognitive testing were as follows: general reasoning (Raven's progressive matrices), $8.6 \pm 1.8 ;$ spatial visualization-visualization of 
TABLE 1. Summary of cognitive test and skills assessment scores for entire cohort

\begin{tabular}{|c|c|c|}
\hline Test & Description & Mean score \\
\hline \multicolumn{3}{|l|}{ Cognitive } \\
\hline Raven's Advance Progressive Matrices & General reasoning test assessing abstract reasoning and pattern recognition & $8.6 \pm 1.8$ \\
\hline Mental Rotation & Spatial visualization test assessing ability to rotate of 3-dimensional objects in space & $51.6 \pm 20.2$ \\
\hline Visualization of Views & $\begin{array}{l}\text { Spatial visualization test assessing ability to imagine } 3 \text {-dimentional objects form } \\
\text { multiple vantage points }\end{array}$ & $12.1 \pm 4.9$ \\
\hline Judgment of Line Orientation & Spatial judgment test assessing visual distractibility (field dependence) & $10.3 \pm 3.6$ \\
\hline Rod and Frame & Spatial judgment test assessing visual distractibility (field dependence) & $26.0 \pm 8.6$ \\
\hline \multicolumn{3}{|l|}{ Technical skills } \\
\hline Needle load & Ability to load needle at appropriate angle & $2.7 \pm 1.3$ \\
\hline Point orthogonal to target & Ability to orient needle orthogonal to target & $3.0 \pm 0.9$ \\
\hline Distortion of cylinder & $\begin{array}{l}\text { Ability to judge spatial confinement and inadvertent distortion of simulation } \\
\text { model tissue }\end{array}$ & $2.6 \pm 0.8$ \\
\hline Choice of backhand forehand & Appropriate choice of forehand or backhand & $2.8 \pm 1.1$ \\
\hline Placement and accuracy along annular plane & Ability to accurately place needle along mitral valve annulus & $3.0 \pm 1.2$ \\
\hline
\end{tabular}

Data presented as mean \pm standard deviation.

viewpoints, $12.1 \pm 4.9$, mental rotation test, $51.6 \pm 20.2$; and spatial judgment (field dependence)-Judgment of Line Orientation test, $10.3 \pm 3.6$, Rod and Frame test, $26.0 \pm 8.6$ (Table 1). In all cases, the scores were normally distributed.

\section{Technical Skills Assessment}

The results from the skills assessment demonstrated a normal distribution. The mean scores for each parameter assessed were as follows (Table 1): NL, $2.7 \pm 1.3$; point orthogonal to target, $3.0 \pm 0.9$; distortion of cylinder, 2.64 \pm 0.81 ; choice of forehand or backhand, $2.8 \pm 1.1$; and placement and accuracy along annular plane, $3.0 \pm 1.2$.

\section{Relationship Between Cognitive Test Scores and Skills Assessment}

Significant correlations were found between the 2 spatial judgment tests (Judgment of Line Orientation and Rod and Frame tests) and the NL skill measure (Table 2). Specifically, the correlations with the NL in an inverse fashion $(r=0.61, P<.05$, and $r=-0.52, P=.05$, Judgment of Line Orientation test and Rod and Frame test, respectively) suggested that residents who were more field independent (not influenced by environmental distractors) performed better vis-à-vis the anticipation and execution of needle angle loading (Figure 4). The NL did not correlate significantly with any spatial judgment tests, suggesting that it is the ability to make spatial judgments, regardless of the distractors (consistent with field independence), that is the important variable for this skill, not the broader ability to mentally manipulate spatial information.

The residency training level did not correlate with any of the cognitive testing scores (Table 2, last row). However, the rating scores of 2 of the 5 skills demonstrated correlations with the resident training level. Specifically, the NL and the appropriate choice of backhand or forehand correlated with the postgraduate year of training $(r=0.56$ and $r=0.58$, respectively; $P<.05$ ), consistent with the ability of the assessment to measure skills progression during residency.

\section{DISCUSSION}

To explore some of the cognitive underpinnings of task acquisition, the present study used established cognitive

TABLE 2. Correlation between cognitive tests and skills assessment scores

\begin{tabular}{|c|c|c|c|c|c|}
\hline \multirow[b]{3}{*}{ Skills assessment } & \multicolumn{5}{|c|}{ Cognitive testing area } \\
\hline & \multirow{2}{*}{$\begin{array}{c}\text { General reasoning } \\
\text { Raven's } \\
\text { Matrices }\end{array}$} & \multicolumn{2}{|c|}{ Spatial visualization } & \multicolumn{2}{|c|}{ Spatial judgment and field dependence } \\
\hline & & $\begin{array}{c}\text { Mental } \\
\text { Rotation }\end{array}$ & $\begin{array}{c}\text { Visualization } \\
\text { of Views }\end{array}$ & $\begin{array}{c}\text { Judgment of } \\
\text { Line Orientation }\end{array}$ & Rod and Frame \\
\hline Needle load & 0.13 & 0.31 & -0.09 & $0.61^{*}$ & $-0.52 *$ \\
\hline Point orthogonal to target & 0.00 & -0.07 & -0.12 & 0.06 & 0.09 \\
\hline Distortion of cylinder & -0.24 & -0.37 & -0.29 & 0.07 & 0.08 \\
\hline Choice of forehand or backhand & 0.12 & 0.25 & -0.10 & 0.32 & 0.46 \\
\hline placement and accuracy along annular plane & 0.09 & 0.10 & -0.19 & 0.09 & 0.12 \\
\hline Postgraduate year & -0.34 & -0.22 & -0.38 & -0.01 & 0.11 \\
\hline
\end{tabular}

Data presented as Pearson's correlation coefficients. Positive scores on the Judgment of Line Orientation and Rod and Frame tests correlated significantly with the ability to anticipate correct needle loading onto the needle driver. *Statistically significant correlation. 


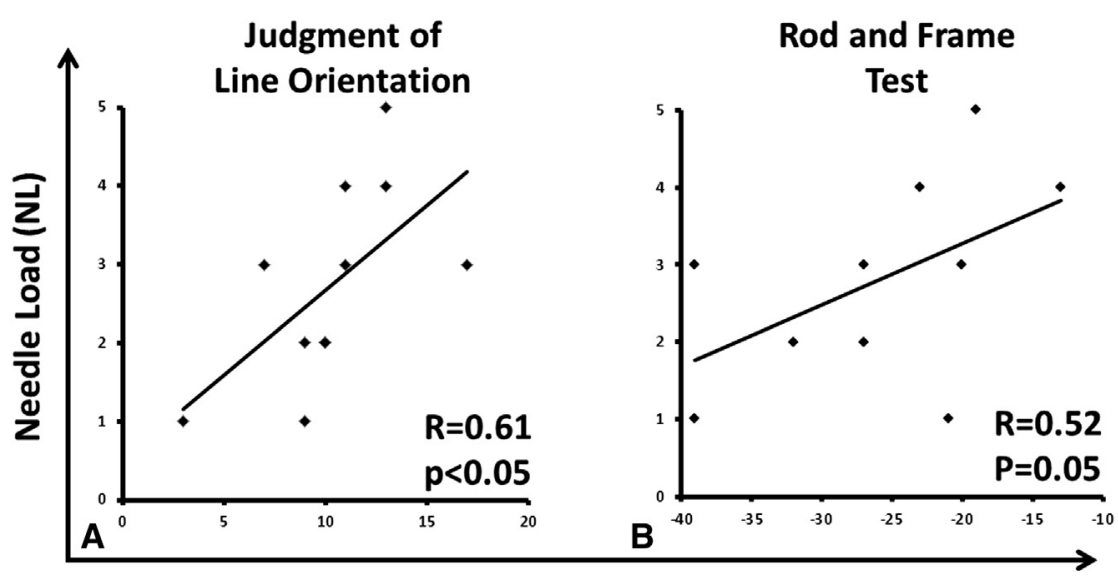

Field Independence

FIGURE 4. Correlation scatter plots demonstrating positive correlations between the individuals' ability to anticipate the proper needle angle loads and the (A) Judgment of Line Orientation and (B) Rod and Frame cognitive spatial judgment tests. For both graphs, the y-axis demonstrates an increasing needle loading skill score and the $\mathrm{x}$-axis increasing field independence.

tests that assessed spatial judgment and spatial visualization skills. Our preliminary findings suggest that the cognitive trait of field independence (characterized as the ability to make accurate spatial judgments even in the presence of distracting elements in the field) might be linked to how individuals determine the appropriate needle angles and NL in confined spaces. Specifically, individuals classified as field independent appeared to have an improved ability to judge appropriate NLs at the correct angles in a small, confined space. Although our data were from a small cohort and observational, our findings lay a premise for additional investigations of the cognitive processes by which surgical trainees might learn new tasks. Directed communication about these processes between educator and resident and the development of training tools to improve our understanding of the effect of frame dependence could eventually lead to improved teaching methods.

Several types of spatial cognitive processes have been identified-spatial memory, spatial perception, spatial visualization, spatial orientation, and so on. ${ }^{11}$ Thus, rather than simply concluding that "spatial ability" is important for surgical skill, it would be potentially more valuable if we could tease apart the particular spatial processes involved in a given skill. Making these explicit would allow both trainers and trainees to become more aware of the particular processes they are engaging at any moment and to identify methods to target the key processes for assessment or intervention during training. This might practically translate into a graded approach to teaching suturing in the simulation laboratory, for example. If a particular trainee were very visually distractible (field dependent), it might be more valuable for that trainee to spend time practicing needle manipulation in a relatively quiet and uncluttered surgical field, before adding increasing levels of complexity. Examples of increasing complexity might include the gradual addition of cannula, suction devices, blood, and movement (eg, beating heart or inflating lungs) using high fidelity simulators such as that described by Ramphal and colleagues. ${ }^{12}$

Although the relationship between needle angle ability and field dependence was clear in the present investigation, other trends merit discussion. For example, the choice of backhand or forehand by the resident also correlated strongly with field dependence, albeit not significantly. Globally, the choice of backhand or forehand represented whether the surgeon made an appropriate choice (and level of hesitation) before executing the movement. This is a more binary skill than the needle angle load; thus, it was likely that the range in the technical skill was not enough to allow for more subtle correlations with frame dependence to manifest. Future studies with a larger sample size will be important to ascertain whether field dependence is significantly related to overall needle management, including the choice of forehand and backhand. If our hypothesis is correct, we predict that field dependence will likely be related to global needle management, including the forehand or backhand choice, because the surgeon must accurately judge the area to be sutured, despite distracting elements in the operative field.

As expected, certain skills assessment scores correlated positively with the trainee level of experience (or postgraduate year). However, the relationship between accurately predicting the needle angles and degree of frame dependence was independent of the resident training level. Thus, more experienced residents were not more frame independent than those with less experience. This supports established data that frame dependence might be an inherent trait. Moreover, these findings suggest that although frame dependence can influence how individuals will predict needle angles and work in 3D spaces, it is 
only part of a much greater cognitive construct required to perform tasks. This might explain why frame-dependent individuals with a greater amount of surgical training were able to perform well on the skills testing. Additional study is required to determine what value frame dependence carries in relation to an individual's overall experience or training level.

\section{Study Limitations}

In the present study, we obtained only a snapshot of visual spatial skills and the relationship to surgical performance in a small cohort at 1 point in time, rather than a longitudinal trace of this ability over time. ${ }^{8,13}$ Therefore, more robust studies are necessary to determine how field dependence might influence the acquisition of surgical skills over time and with increased experience. Concerns could exist with the validity of our measures; administering the adapted Rod and Frame test on paper requires fewer resources and allows group testing, but it might have resulted in a somewhat different conceptualization of this construct, which hinged on judgments related to true vertical and thus had a larger "self-orientation" component. A validation study of the paper version and the original version would determine whether they assess the same components of spatial ability. In addition, concern could exist for a "learner effect" regarding the subjects' performances on the cognitive and skills testing and bias on the part of the expert reviewers, given they were faculty at our institution. In a retrospective review, none of the subjects had reported participating in the administered exercises previously; thus, the learner effect was likely minimal in our cohort. Finally, the findings from simulation-based studies of learning and skills training in cardiac surgery might not transfer to the operative setting; ensuring transferability will require defining the tasks that can be simulated and that can be accurately assessed in the operating room without affecting patient safety.

\section{CONCLUSIONS}

The results of our study suggest that field independence, as measured by the Judgment of Line Orientation test and adapted Rod and Frame test, might relate to a specific technical skill (needle loading), separate from the broader cognitive spatial visualization ability. These findings require additional study and validation before clinically relevant and practical training tools can be developed. The concept of field independence should be investigated as one of the many aspects that contribute to the learner's composition, such that individually tailored training regimens can be designed to best meet the needs of our surgeons-in-training. Eventually, trainers will be able to drawn on tools that combine expertise from both the clinical teaching realm and the fields of cognitive psychology to train learners more efficiently and gauge their progress over time.

We gratefully acknowledge all the residents and Stephanie Harrington for their assistance with the present study.

\section{References}

1. Issenberg SB, McGaghie WC, Petrusa ER, Lee Gordon D, Scalese RJ Features and uses of high-fidelity medical simulations that lead to effective learning: a BEME systematic review. Med Teach. 2005;27:10-28.

2. Reznick RK, MacRae H. Teaching surgical skills_changes in the wind. $N$ Engl J Med. 2006;355:2664-9.

3. Tse AM, Iwaishi LK, King CA, Harrigan RC. A collaborative approach to developing a validated competence-based curriculum for health professions students. Educ Health (Abingdon). 2006;19:331-44.

4. Joyce DL, Dhillon TS, Caffarelli AD, Joyce DD, Tsirigotis DN, Burdon TA, et al. Simulation and skills training in mitral valve surgery. J Thorac Cardiovasc Surg. 2011;141:107-12.

5. Fann JI, Feins RH, Hicks GL Jr, Nesbitt JC, Hammon JW, Crawford FA Jr, et al. Evaluation of simulation training in cardiothoracic surgery: the Senior Tour perspective. J Thorac Cardiovasc Surg. 2012;143:264-72.

6. Fann JI, Caffarelli AD, Georgette G, Howard SK, Gaba DM, Youngblood P, et al. Improvement in coronary anastomosis with cardiac surgery simulation. J Thorac Cardiovasc Surg. 2008;136:1486-91.

7. Zacks JM, Michelon P. Transformations of visuospatial images. Behav Cogn Neurosci Rev. 2005;4:96-118.

8. Keehner MM, Tendick F, Meng MV, Anwar HP, Hegarty M, Stoller ML, et al. Spatial ability, experience, and skill in laparoscopic surgery. Am J Surg. 2004; 188:71-5.

9. Kozhevnikov M. Cognitive styles in the context of modern psychology: toward an integrated framework of cognitive style. Psychol Bull. 2007;133: 464-81.

10. Cote CA, O'Donnell AM. Field dependence and stimulus complexity in a figure copying task. Perceptual Motor Skills. 2007;105:1159-70.

11. Hegarty M, Waller DA. Individual differences in spatial abilities. In: Shah P, Miyake A, eds. The Cambridge Handbook of Visuospatial Thinking. New York: Cambridge University Press; 2005:121-69.

12. Ramphal PS, Coore DN, Craven MP, Forbes NF, Newman SM, Coye AA, et al. A high fidelity tissue-based cardiac surgical simulator. Eur J Cardiothorac Surg. 2005;27:910-6.

13. Keehner M, Lippa Y, Montello DR, Tendick F, Hegarty M. Learning a spatial skill for surgery: how the contributions of abilities change with practice. Appl Cogn Psychol. 2006;20:487-503. 